\title{
WEEE collection and CRM recovery trials: piloting a holistic approach for Scotland
}

\author{
Hursthouse A. ${ }^{1,}{ }^{*}$, Kelly S. ${ }^{1}$, McPherson W. ${ }^{3}$, Menzies B. ${ }^{4}$, Mirzaeian M. ${ }^{2}$, Wood D. ${ }^{4}$, Hendry S. ${ }^{1}$ and Abbas Q. ${ }^{2}$ \\ ${ }^{1}$ School of Science \& Sport, University of the West of Scotland, Paisley, PA1 2BE UK \\ ${ }^{2}$ School of Engineering \& Computing, University of the West of Scotland, Paisley, PA1 2BE UK \\ ${ }^{3}$ Re-Tek UK, East Kilbride, G75 OAP UK \\ ${ }^{4}$ Enscape Consulting, Stonehaven, AB39 $2 \mathrm{HX}$ UK \\ Received: 25/02/2018, Accepted: 01/05/2018, Available online: 26/09/2018 \\ *to whom all correspondence should be addressed: e-mail: andrew.hursthouse@uws.ac.uk \\ https://doi.org/10.30955/gnj.002643
}

\section{Abstract}

Re-Tek UK and its partners, Enscape Consulting and the University of West of Scotland commenced trials for the collection and recovery of critical raw materials from waste electrical and electronic (WEEE) products in July 2016. Sponsored by the EU LIFE funded project 'Critical Raw Material Closed Loop Recovery' coordinated by WRAP with EARN, ERP UK Ltd, KTN Ltd and Wuppertal Institute as beneficiaries. The trials are aimed at boosting the recovery of critical raw materials (CRMs) from household waste electrical and electronic products (WEEE) and Information Communications Technology (ICT) in particular, after functioning equipment is separated out for re-use.

The new collection models provided residents with the opportunity to drop-off unwanted electrical and electronic appliances at a time and place that suits them, through a collaborative approach which encourages local authorities, educational establishments, businesses, and Social Enterprises, etc to act as hub sites. Hubs were designed to minimize product damage and encourage drop-off, rather than hoarding.

Extraction methods developed after the collection phase of the trial looked at the opportunity to recover cobalt, gold and silver from ICT products, with the potential to inform how a more sustainable supply chain could be developed in Scotland. The elements studied were selected to demonstrate financial opportunity (gold/silver) and a strategic priority material (cobalt) for long term supply. These are based on bioleaching and electrochemical recovery using novel carbon based electrode systems, and chemical processing methods using extraction techniques with an assessment of pilot performance and scale up challenges. Our report is on the state of progress towards practical solutions to WEEE and CRM recovery.

Keywords: Critical raw materials, bioleaching, chemical extraction, electrochemical recovery, electronic equipment collection, hub sites, cobalt, silver, gold.

\section{Introduction}

Waste Electrical and Electronic Equipment (WEEE) is currently considered to be one of the fastest-growing waste streams in Europe. Currently, the world generates around 40 million tonnes of e- waste every year (Balde et al., 2015). The EC estimates that in weight terms only $30 \%$ of WEEE is appropriately collected and recycled (EC, 2008). The remainder goes to landfills (13\%) and potentially for substandard treatment inside or outside the EU. Currently approximately $2 \%$ of WEEE is reused but EC estimates are that $5 \%$ of collected WEE is suitable for reuse (EC, 2008) E-waste is of serious concern due to the loss of valuable Critical Raw Materials (CRMs) which are also often hazardous materials, with the potential for significant impacts, which must be mitigated against, if disposed of to landfill or incinerated through informal, illegal waste exchange. The environmental impacts of disposal practices across the world are often most severe in developing countries, where vulnerable communities are often most at risk.

Demand for raw material, in particular for precious metals or CRMs, is growing due to their value to a range of different manufacturing sectors and on-going concerns regarding the security of their supply.

The Critical Raw Materials Closed Loop Recovery project is delivering a series of collection and recovery trials across 4 European countries that consider the impact of collection on reuse and recovery potential on WEEE and materials it contains. The information and evidence gathered through the trials will support the development of a European wide infrastructure plan and policy recommendations (EC, 2008) to support the increased recovery of critical and valuable materials from WEEE. By evidencing the potential of collection and recovery techniques this project has the ability to impact the industry by increasing the availability of critical raw materials (CRMs) for use in new products. This study forms one of a number of trials being developed through EU LIFE funded Critical Raw Material Closed Loop Recovery project, coordinated by WRAP (UK) (WRAP, 2016). The structure of the case study is summarised in the schematic below (see Figure 1). 


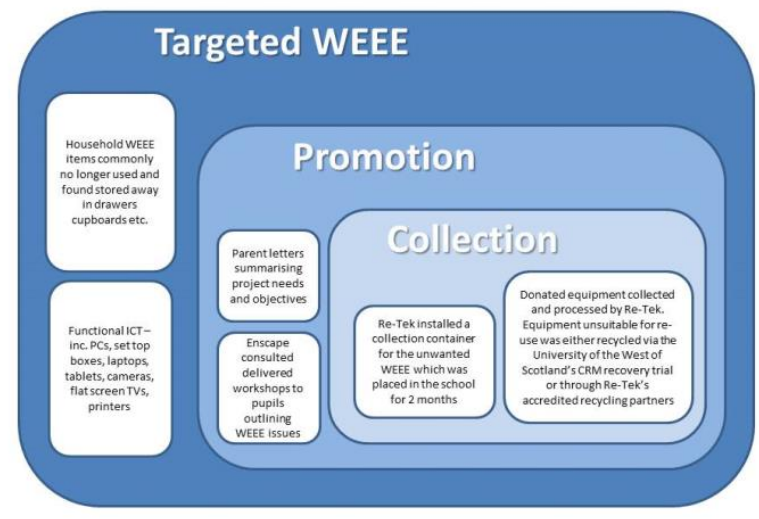

Figure 1. Schematic overview of trial process (based on WRAP (2016))

The outcomes from our trial will provide information and data to inform:

- $\quad$ The type and quantity of equipment that can be collected from a number of different collection/logistics models. From this, equipment which has been tested, refurbished and wiped of data has been sold into domestic and international markets. The residual equipment, considered suitable for reprocessing was identified, with Printed Circuit Boards (PCBs), given the high concentration of components selected for the innovative reprocessing techniques forming part of Phase 2.

- How ICT WEEE that cannot be reused can be responsibly recycled using a method that maximizes CRM recovery cost effectively and safely.

- How current and evolving policy and reuse/recycling infrastructure status is able to facilitate the delivery of the vision e.g. through regulations, good practice, systems, economics etc.

\section{Methods}

The project was time and resource limited and was split into two phases to maximise breadth of process evaluation, steps undertaken were as summarized below:

- $\quad$ Phase 1: Equipment collection, using the different collection/logistics models

- $\quad$ Phase 2: Selected PCBs prepared and processed using chemical, biological and electro-chemical methods - for the extraction of Critical Raw Materials (CRMs).

The collection models trialled by the Re-Tek-led team were developed to provide consumers with the opportunity to drop-off unwanted electrical and electronic appliances at a time and place that suited them, to reduce hoarding of potentially valuable resources. The approach was a collaborative one aimed at incentivizing local authorities, educational establishments, businesses, and social enterprises etc to act as hub sites. These hubs were also designed to minimize product damage and encourage drop-off. Secure receptacles were provided at each location to ensure integrity of collection and isolate hazardous materials. The type of equipment being sought was functional ICT as listed in Table 1:

Table 1. WEEE component collection categories

\begin{tabular}{cc}
\hline Laptops & $\begin{array}{c}\text { Miscellaneous Audio/Video } \\
\text { Devices }\end{array}$ \\
\hline Desktop/Inkjet Printers & Personal computers \\
\hline $\begin{array}{c}\text { Compact Digital } \\
\text { Cameras }\end{array}$ & Tablets \\
\hline Computer Components & Flat Screen Monitors \\
\hline Networking Items & Flat screen Televisions \\
\hline Digital Set Top Boxes & Mobile Phones \\
\hline Gaming Devices & New Printer Cartridges \\
\hline
\end{tabular}

The Phase 1 collections models involved establishing the following:

- $\quad$ Re-use containers at Household Waste Recycling Centers (HWRCs)

- Employee amnesty collections (business-tobusiness, B2B)

- Schools as collection hubs

- University Halls of Residences as collection hubs (Halls)

- Social economy organizations, as collection partners.

Phase 2 involved the set- up of novel laboratory based investigations for the recovery of gold, silver and cobalt from WEEE in an electrochemical flow system consisting of a series of electrochemical cells. The method of metal recovery included electrodeposition in which metal ions in the treatment solution (obtained by the dissolution of shredded WEEE in aqueous acidic solution) recovered at the cathode where the positive metal ions in the solution migrate and are deposited as high purity metal. The trials were undertaken on PCBs collected at Re-Tek through C2B recovery, where the age and origin of the boards could be recorded.

The potential of microbial biomass to solubilize/mobilize specific metals from WEEE as a pre-treatment or hybrid recovery methodology was also examined by Bioleaching. This included solid/particulate WEEE pre-treatment in agitated mini-reactors (flasks) under controlled conditions (dependent on microbial biomass used) and performance analysis of the process (by atomic spectroscopy ICP-OES/MS), including the analysis of filtered liquid culture media after microbial incubation with solid WEEE samples.

A series of chemical extraction techniques which included acid leaching and chemical precipitation (sulphide and organic reagent reduction based). Each trial was undertaken as a discrete batch experiment to assess the feasibility of treatment using the individual approach.

The methods trialled are summarised here: 


\subsection{Physical preparation}

Particle size analysis was carried out to determine whether grinding would aid the separation of metal concentrations in the PCB boards. With a particular emphasis based on the removal of high copper content and gold which overlap on the electrochemical cell. The PC's from which the PCB's were selected from, were all of a similar age and the medium age in all of the PCB's available for analysis.

PCB pieces below $2 \mathrm{~mm}$ in size were placed in a Retsch RM200 lab electrical mortar grinder and then ground down for 10 minutes, to form a fine powdered sample. The sample was then sieved into the different particle sizes $<106 \mu \mathrm{m}, 106-250 \mu \mathrm{m}, 250-500 \mu \mathrm{m}$ and $500 \mu \mathrm{m}-2 \mathrm{~mm}$ particle sizes.

\subsection{Biological separation}

Three different types of microbe were selected (acidophiles, fungus and cyanogenic) to determine whether they would selectively extract gold, silver and cobalt from ground PCBs. Mixed cultures of cyanogenic bacteria Chromobacterium violaceum and Bacillus megaterium in agitated culture flasks $(500 \mathrm{ml})$ were investigated for the recovery of critical resource materials from waste printed circuit boards (WPCBs) originating from mobile phones, notebooks and desktop personal computers.

To remove solder mask and improve microbial access to metals WPCBs were soaked in $\mathrm{NaOH}(10 \mathrm{M})$ for 24 hours before washing with distilled water until neutral $\mathrm{pH}$ of wash water was confirmed (Jadhav and Hocheng, 2015). WPCBs were added to flasks 2 days after inoculation to alleviate toxic effects of e-waste on biomass growth and cyanide formation (Natarajan and Ting, 2015). Following incubation, cultures were centrifuged to separate biomass from leach liquor. Acid digestion and filtration of leach liquor and retrieved-WPCBs was carried out. Biomass was lysed using aqua regia and subsequently filtered. Analysis by ICP-MS was used to determine mass balance of metals in leach liquor, associated with biomass and remaining on WPCBs after day 17.

In a second study acidophilic pre-treatment with Acidithiobacillus ferrooxidans (7 days) was followed by a subsequent cyanogenic incubation with Bacillus megaterium (4 days). Following both acidophilic and cyanogenic stages cultures were processed to recover metals for analysis.

A third study involved the leaching with Aspergillus Niger which was grown on malt extract agar at $28^{\circ} \mathrm{C}$ for 5 days, harvested and placed in flasks with PCB samples at different stages of degradation and to assess the leaching potential of exudates form the organisms..

\subsection{Chemical separation}

Four chemical separation techniques were explored to extract gold, silver and cobalt, these were i) acid dissolution (aqua regia and other systems), ii) sulphide precipitation using standard Kipps apparatus and acid generation of $\mathrm{H}_{2} \mathrm{~S}$ from iron sulphide powder, and iii) particle size distribution. The fourth extraction method which used hydroquinone to extract solely gold was also considered. Based on historical preparation methods (Beamish et al., 1937), this study looked at hydroquinone as a reducing agent for precipitation of gold from leached solutions and standard materials as a preliminary evaluation step in process development.

\subsection{ElectroChemical cell}

Fourteen proprietary EC Cells were manufactured for the recovery of gold, silver and cobalt from reference solutions. These were created using polymeric gel by solvent exchange, controlled drying and selection of candidate gels for carbonisation. The carbonised gels were then characterised by nitrogen adsorption and successful carbon materials used in electrochemical recovery experiments. Reference solutions of metals of interest were then used to study recovery rates at fixed potentials.

\section{Results and discussion}

Summary data for the material collected from the various collection models is shown in Figure 2. This identifies numbers of items collected and includes an indication of any subsequent reuse post collection as well as an age distribution of items recovered. Total weight of items from each collection method is also identified (see Table 2). In summary:

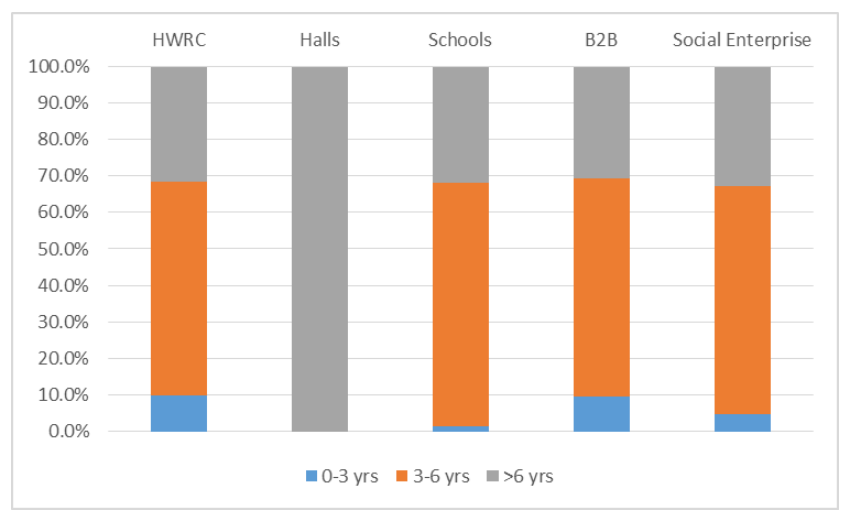

Figure 2. WEEE recovery data: items collected \% by age from each collection method

a) The outcome of Phase 1 collection model trials indicates just over $90 \%$ of the pre-project estimates of equipment that would be collected, were actually collected (the former based on industry benchmarking suggesting for B2B and C2B models 900 items would be recovered).

b) The levels of re-use achieved from all of the collection models trialled are significantly less than is often found to be the case from mainstream, ongoing business to business contracts (which typically exceed $80 \%$ ). Re-use levels ranged from $27 \%$ for equipment donated through HWRCs to $30 \%$ and $36 \%$ for equipment donated through schools and Social Enterprises, respectively. There are considered to be many reasons for such low levels, one of which is that equipment donated appears to be older. 
An average of $8.6 \%$ of equipment (across all collection models) was between 0 and 3 years old, with $59.7 \%$ in the range of 3 to 6 years. B2B clients, given the average IT re-fresh programs of three years, demonstrate circa $20 \%$ between $0-3$ years with the balance, circa $80 \%$, between $0-5$ years.

c) The age range of equipment collected as a whole did not vary significantly for the different collection models. However, when different categories of equipment were considered there were significant difference in age ranges and levels of re-use. $95.7 \%$ of all the equipment collected in Phase 1 fell under two categories: ICT (71\%) and TVs and monitors (24.7\%). HWRCs resulted in $11.5 \%$ re-use, with School and B2B collections more than double this $(25.4 \%$ and $23.4 \%$ respectively).

d) HWRCs provided a significantly lower percentage of ICT than the other models $-64 \%$ compared to between $73 \%$ and $89 \%$. A significant contribution to the HWRC re-use rate, overall, was related to flat screen TVs and monitors. Flat screen TVs and monitors may represent a potential opportunity for HWRCs due to the relatively higher reuse rates and revenue generated. Increasing numbers of HWRCs are already providing separate containers for screens (for recycling). The approach to collecting ICT separately needs further consideration due to what appears to be a reluctance amongst consumers to donate high value data-bearing devices to HWRCs.

e) Significant differences in the types of ICT equipment collected were seen, for the different collection models e.g. $42.4 \%$ of HWRC and $38.3 \%$ of Social Enterprise WEEE were printers. This compares to between $3.4 \%$ and $11.5 \%$ for Schools and B2B collections.

f) Considering the ICT category alone (smartphones, mobile phones, tablets, PCs, laptops) there were significant differences in the profile of equipment depending on the collection model employed. $50 \%$ and $53 \%$ of the ICT collected through HWRCs and Social Enterprises respectively was Core ICT, while $73 \%$ of the ICT collected from Schools and B2Bs were ICT. HWRCs and the Social Enterprise collections included significantly higher levels of printers (>40\%) which have very little, if any, re-use/resale potential.

g) The ability to generate viable re-use income streams from Consumer to Business (C2B) collections, from end users, is more challenging than for B2B, and will continue to be the case unless equipment hoarding is minimized, a situation which is likely to mean that enhanced versions of the collection models trialled are developed, and/or alternative approaches are taken forward in the future.
Table 2. summary of collection trial results (weight collected and portion re-used

\begin{tabular}{cccc}
\hline \multirow{2}{*}{$\begin{array}{c}\text { Collection } \\
\text { Model }\end{array}$} & $\begin{array}{c}\text { Weight, } \\
\text { Kg }\end{array}$ & $\begin{array}{c}\text { Total Collection } \\
\text { Collected (\%) }\end{array}$ & $\begin{array}{c}\text { Items Re- } \\
\text { used (\%) }\end{array}$ \\
\hline HWRC & 4,243 & $409(100.0 \%)$ & $111(27 \%)$ \\
\hline Halls & 0.4 & $4(100.0 \%)$ & $0.00(0 \%)$ \\
\hline Schools & 190 & $66(100.0 \%)$ & $20(30 \%)$ \\
\hline B2B & 1,331 & $286(100.0 \%)$ & $76(27 \%)$ \\
\hline $\begin{array}{c}\text { Social } \\
\text { Enterprise }\end{array}$ & 621 & $64(100.0 \%)$ & $23(36 \%)$ \\
\hline TOTAL & 829 & $829(100.0 \%)$ & $230(28 \%)$ \\
\hline
\end{tabular}

The collection of individual PCBs from equipment identified in phase 1 presents the input feed to Phase 2. The preparation of material requires further processing, primarily in size reduction using mechanical shredding in a manner that is viable for bulk waste processing. Size reduction introduces greater surface area for bioleaching and digestion of the solid phase but the process also generates issues of dust production, fractionation of metal phases and inhomogeneous residues (Oguchi et al., 2012). Plastic and metal components perform variably under mechanical treatment and introduce high heterogeneity into shredded product (Ruan and Zum, 2016). A systematic analysis of a number of commercial shredding facilities was undertaken and "best" option identified based on preliminary trials. Shredded test samples were assessed for particle size range. Further impact on phase 2 was addressed through replicate digests for both total metal and bioleached. The scope of the study does not allow optimization or development of full protocol for preparation but highlights some confounding factors. Physical treatment through conventional shredder systems suggests the majority ( $>70 \%$ ) of shredded material falls into 1.5-9 mm particle diameter. A minor proportion of the total material ( 8\%) was less than $1.5 \mathrm{~mm}$.

\subsection{Biological separation}

Metal recovery efficiency by the mixed culture of cyanogenic bacteria varied significantly across different WPCB types and metals; Co (76.1\%), Cu (27.30\%), Ag (7.43\%). Biorecoveries of $\mathrm{Mg}$ up to $76 \%$ was observed which could have a variety of industrial applications. Gold was not biorecovered in this study.

In the second study acidophilic pre-treatment with Acidithiobacillus ferrooxidans resulted in significant recovery of $\mathrm{Au}(70 \%)$, Co (96\%) and $\mathrm{Cu}$ (99\%) with limited additional recovery in the subsequent incubation with Bacillus megaterium. From this study bioleaching appeared to be the primary mechanism for recovery of Au while biosorption or bioaccumulation was the primary mechanism for $\mathrm{Co}$ and $\mathrm{Cu}$ since the latter metals were found to be associated with the biomass rather than the culture medium.

Some abiotic control vessels showed significant recovery of metals from culture medium and require further investigation to further elucidate mechanisms involved. 
The results from the initial Aspergillis Niger microbial leach, were inconclusive and the laboratory work would need to be repeated in order to determine the effectiveness of the microbes.

For the 2-step process, the acidophilic treatment with A. ferrooxidans resulted in significant recovery of Au (up to $70 \%$ ), Co (up to $96 \%$ ) and Cu (up to 99\%) with minor additional recovery in the subsequent cyanogenic stage. The results indicate that gold was typically bioleached into solution, whereas Cobalt and Copper were associated with the biomass (biosorption/bioaccumulation) rather than in solution.

Whilst these results, indicate some promising findings, the abiotic controls were elevated and potentially impact on measured recovery rates.

\subsection{Chemical separation}

\subsubsection{Digestion}

Several chemical digestion procedures were employed on PCB's to assess the feasibility and the success of these digestion methods, in order to provide a reliable and robust characterisation for WEEE waste. Hydrogen Peroxide with Aqua Regia digestion at $95^{\circ} \mathrm{C}$ was determined to be the optimum digestion method for WEEE waste.

\subsubsection{Particle size}

Copper concentrations were shown to increase with increasing particle size while gold concentrations decreased with increasing particle size. Multi elemental analysis has been carried out on the ICP-MS with similar results for metals decreasing and increasing with particle size.

\subsubsection{Sulphide precipitation}

The $\mathrm{pH}$ of optimum precipitation varied for all elements studied. The optimal recovery was between 50 and $96 \%$ and varied across $\mathrm{pH}$ 1-12 due to solubility differences between elements. This provides useful strategy for selective extraction of the elements.

\subsubsection{Hydroquinone extraction}

A common gold extraction method is to precipitate the gold by adding a precipitant to the gold leach solution (Beamish et al., 1937). This causes the gold ions to be reduced and accumulate into larger particles that fall out of solution, this gold precipitate can now be easily filtered and the gold recovered. The chosen gold extraction method avoids the use of cyanide and instead uses a $5 \%$ Hydroquinone solution as a reducing agent under acidic conditions to single out gold ions for reduction to metal. This extraction method is a simple, inexpensive approach that is extremely rapid, with the gold being easily filtered, However further analysis and method development is required to ensure elemental purity when treating multi element printed circuit boards.

- Extracting gold using hydroquinone was relatively straightforward with promising recovery levels in the first assessments.

Particle size distribution analysis was undertaken to establish of this could be an aid in establishing whether specific types of metals accumulated in particular size ranges of $P C B$ after shredding e.g. showing CRM-rich areas of the PCB.

Multi-elemental analysis showed that gold and cobalt increased in concentration with decreasing particle size and thus there may be the potential for physical separation from $\mathrm{Cu}$ and $\mathrm{Zn}$ which increase in concentration with increasing particle size. The results for silver were inconclusive.

\subsection{Potentiostatic electrodeposition using electrochemical cell}

Carbon electrodes with controlled porosity and specific surface area were prepared for the electrodeposition of the metal ions from model solutions using electrochemical cell (EC) designed in the lab. The EC Cells were tested using model solutions, made up to a known concentration. This enabled accurate recovery rates to be determined. The recovery of target metals from model solutions was performed by optimizing the applied potential and the scan rate using cyclic voltammetry (CV) technique. Different potential ranges were used depending on the type of metals to be recovered.

1) Gold recovery was performed in the potential range of -1000 to $-500 \mathrm{mV}$ whereas the optimized scan rate of $1 \mathrm{mV} / \mathrm{s}$ was used.

2) Operating potential of -500 to $700 \mathrm{mV}$ was used for the recovery of silver using scan rate of $1 \mathrm{mV} / \mathrm{s}$.

3) Improved recovery of cobalt was achieved using $0.5 \mathrm{mV} / \mathrm{s}$ scan rate in the potential window of -1000 to $-700 \mathrm{mV}$.

All the electrochemical analysis was performed below the maximum potential of $1.23 \mathrm{~V}$ to avoid the decomposition of aqueous solutions. (Yang, 2003; Poisot-Díaz et al., 2008). Potentiodynamic curves of working carbon electrodes during the recovery of cobalt, gold and silver using electrochemical cell are shown in Figure 3. Gradual increase in current density with increase in applied potential can be observed on all voltammograms. This can be attributed to the current produced due to the electrodeposition of metallic ions when applying electric potential in a range corresponding to the potential window of each metal (Freitas and Garcia, 2007). Oxidation peaks around -0.9 and $-0.7 \mathrm{~V}$ represents the removal of cobalt from the solution while oxidation peaks around $-0.95 \mathrm{~V}$ and $0.3 \mathrm{~V}$ represent the elimination of gold and silver ions from the solutions and their electrodeposition onto carbon electrodes respectively (Devi et al., 2016).

The results utilising the reference solutions appear promising, with gold, silver and cobalt recovery rates of up to $99 \%, 95 \%$ and $98 \%$ respectively. The cells appear to be effective at recovering these metals at concentrations as high as $1000 \mathrm{ppm}$ and as low as $1 \mathrm{ppm}$. There appears to be some contamination arising from the EC Cell itself, but the cause is currently unclear and requires further investigation. 

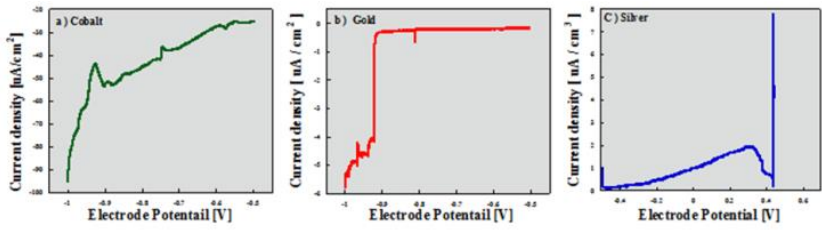

Figure 3. Cyclic Voltammograms (CV) obtained during electrodeposition of various metal ions (a) electrodeposition of cobalt at the scan rate of $0.5 \mathrm{mV} / \mathrm{s}$ (b) electrodeposition of gold at the scan rate of $1 \mathrm{mV} / \mathrm{s}$ (c) electrodeposition of silver at the scan rate of $1 \mathrm{mV} / \mathrm{s}$

\section{Conclusions}

a) The Phase 1 collection trials may indicate that consumers prefer to donate potentially data bearing equipment to schemes that are embedded within the community e.g. through schools and social enterprises, however a detailed investigation into consumer motivations, to donate/hoard equipment would be useful to target future campaign materials and refine collection schemes to maximize newer, higher value equipment.

b) Consider maintaining support and working closely with local social enterprises and schools, which have a local dimension, and have a trusted relationship with the local community. With little in the way of infrastructure required to develop such relationships, the main risks to consider are the cost of staff time to support this. Such relationships may also provide benefits in terms of wider appeal to the community, and access to additional funding revenues which may enable cost-effective schemes to be delivered. In addition, associated with such initiatives would be the wider social benefits of providing work and volunteering opportunities to vulnerable members of the community, doing so within a circular economy context.

c) There would appear to be an opportunity to target flat screen TVs/monitors at HWRCs, since at this point in time they are not perceived to be data bearing equipment in the way that laptops, PCs, tablets and smartphones are. Many HWRCs already segregate screens, and targeting flat screen TVs and monitors for reuse may be a particularly viable collection opportunity. Targeting larger items such as flat screen TVs and monitors may also help to change the culture of hoarding due to the amount of space required to store these items in the home and encourage consumers to view the end of life of all devices differently.

d) The compliance scheme model, and how it supports Social Enterprises, is an area that could be considered further, in terms of how it evolves to support collaboration and greater income sharing among these potentially effective delivery partners in the future.

e) Discussions have indicated that it is important to gain a good understanding of the contractual status of local authorities and their compliance scheme partners, in terms of the type/quantities of WEEE being removed from HWRCs. Where the contractual circumstances mean that there are opportunities to divert WEEE from recycling, for re-use, there would be benefits in work being undertaken to quantify this and to feed it back so that the scale of the opportunities are understood.

f) Biological and Chemical treatments show good potential but are severely limited at this stage by variability in the feed stock for treatment. Analytical methods for metal determinations are still under development and validation is needed. The distribution between physical size fractions and inhomogeneity confounds some of the results, but at this stage there are promising options to study new hydrometallurgical methods with and without biological treatment systems. Areas for development and review would include the potential for enhancing bioleaching processes. The investigation of new microbial consortia, biocatalytic activity of potential additives and a full assessment of the implications for waste generation from leaching process would follow.

\section{Acknowledgements}

This study was sponsored by the EU LIFE funded project 'Critical Raw Material Closed Loop Recovery' coordinated by WRAP with EARN, ERP UK Ltd, KTN Ltd and Wuppertal Institute as beneficiaries.

\section{References}

Balde C.P., Kuehr R., Blumenthal K., Fondeur Gill S., Kern M., Micheli P., Magpantay E. and Huisman J. (2015), E-waste Statistics: Guidelines on Classifications, Reporting and Indicators. United Nations University, IAS - SCYCLE, Bonn, Germany. 2015.

Beamish F.E., Russell J.J. and Seath J. (1937) The Determination of Gold, Industrial \& Engineering Chemistry, 9(4), 174-176.

Devi P., Bansod B., Kaur M. Bagchi S. and Nayak M.K. (2016), Coelectrodeposited $\mathrm{rGO} / \mathrm{MnO} 2$ nanohybrid for arsenite detection in water by stripping voltammetry, Sensors and Actuators B: Chemical, 237, 652-659.

EU Commission (2008) http://europa.eu/rapid/pressrelease_MEMO-08-764_en.htm, last accessed 15/02/2018.

Freitas M. and Garcia E. (2007), Electrochemical recycling of cobalt from cathodes of spent lithium-ion batteries, Journal of Power Sources, 171, 953-959.

Jadhav U. and Hocheng H. (2015), Hydrometallurgical recovery of metals from large printed circuit board pieces, Scientific Reports, 5, 14574.

Natarajan G. and Ting Y.-P. (2015), Gold biorecovery from e-waste: An improved strategy through spent medium leaching with $\mathrm{pH}$ modification. Chemosphere, 136, 232-238. 
Oguchi M., Sakanakura H., Terazono A. and Takigami H. (2012) Fate of metals contained in waste electrical and electronic equipment in a municipal waste treatment process, Waste Management, 32, 96-103.

Poisot-Díaz M.E., González I. and Lapidus G.T. (2008), Electrodeposition of a silver-gold alloy (DORE) from thiourea solutions in the presence of other metallic ion impurities, Hydrometallurgy, 93, 23-29.

Ruan J. and Xu Z. (2016) Constructing environment-friendly return road of metals from e-waste: Combination of physical separation technologies, Renewable and Sustainable Energy Reviews, 54, 745-760.

WRAP (2016), Critical Raw Material Closed Loop Recovery, ('CRM Recovery') http://www.criticalrawmaterialrecovery.eu/, last accessed on 15/02/2018.

Yang C.-C. (2003), Recovery of heavy metals from spent $\mathrm{Ni}-\mathrm{Cd}$ batteries by a potentiostatic electrodeposition technique, Journal of Power Sources, 115, 352-359. 